\title{
Decay of high-frequency phonons in amorphous silicon
}

\author{
A. J. Scholten and J. I. Dijkhuis \\ Faculty of Physics and Astronomy and Debye Institute, University of Utrecht, P.O. Box 80.000, 3508 TA Utrecht, The Netherlands
}

(Received 19 June 1995)

\begin{abstract}
We present measurements concerning the dynamics of high-frequency phonons $\left(\hbar \omega \geqslant 150 \mathrm{~cm}^{-1}\right)$ in amorphous silicon at $2 \mathrm{~K}$. From pulsed anti-Stokes Raman experiments, estimates of the population decay time are obtained for high-frequency phonons, generated by fast carrier relaxation. The fracton model explains the counterintuitive sense of the decay-time dependence on frequency in terms of the decrease of the localization length of the vibrational states towards higher frequencies, and the concomitant suppression of the anharmonic decay rate. This model fits our data with a very realistic third-order elastic constant.
\end{abstract}

\section{INTRODUCTION}

In many aspects, amorphous solids differ markedly from their crystalline counterparts. Their vibrational properties, for instance, are characterized by an intriguing set of universal characteristics, only weakly dependent on the specific chemical composition. The low-temperature excess specific heat and the thermal conductivity plateau are the most prominent anomalous properties. ${ }^{1}$ The two-level-system model $^{2}$ successfully accounts for the thermal properties of amorphous materials at the lowest temperatures $(T<1 \mathrm{~K})$, and has recently been extended to higher temperatures in the framework of the soft-potential model. ${ }^{3}$ Despite this progress, there is still no general consensus about the origin of the plateau in the thermal conductivity, and about the mechanism responsible for the thermal conduction above the plateau. A more fundamental question, intimately related to the previous ones, is what happens at high phonon frequencies, when the phonon mean free path approaches the phonon wavelength. The critical point, known as the Ioffe-Regel limit $\omega_{\text {IR }}$, typically occurs at a phonon frequency around $1 \mathrm{THz}$.

Some authors have suggested that phonons with $\omega>\omega_{\text {IR }}$ are localized. ${ }^{4}$ For fractal media, a special class of amorphous materials, a theory has been developed in which localization of the vibrational states above a mobility edge $\omega_{0}$ is indeed a crucial ingredient. In this so-called fracton model, the plateau in the heat conductivity is associated with $\omega_{c}$, and the additional thermal conduction at higher temperatures is explained by thermally activated hopping of localized vibrational states. $^{5}$

Based on computer simulations, however, other authors claim that only a small region is Anderson localized at the high-energy side of the vibrational spectrum of amorphous silicon, ${ }^{6,7}$ and dismiss the identification of the Ioffe-Regel limit with a mobility edge for phonons. ${ }^{7,8}$ They found that the majority of the vibrational eigenstates remain extended above the Ioffe-Regel condition. These vibrations do not propagate, but are thought to carry heat by means of diffusion, as soon as a temperature gradient exists. ${ }^{7,8}$

Experiments on these high-frequency vibrational states have mainly been restricted to studies of the thermal conductivity and the heat capacity. A principal difficulty in interpreting and analyzing these experiments is that the measured quantities always represent a value averaged over a distribu- tion of vibrational states. Spectroscopy of nonequilibrium phonons, however, potentially gives information about the elementary processes associated with vibrational states in amorphous materials.

Recently, we reported pulsed Raman measurements of transient phonon populations in amorphous silicon at $2 \mathrm{~K}$. From the analysis of the excitation-density dependence of the Raman signals, it was concluded that the high-energy ( $\hbar \omega>100 \mathrm{~cm}^{-1}$ ) phonons, emitted as a result of carrier relaxation, possess a nonequilibrium energy distribution. Timeresolved experiments showed some unexpected results. Whereas the $150-\mathrm{cm}^{-1}$ population exhibits a decay that is much faster than $10 \mathrm{~ns}$, the $480-\mathrm{cm}^{-1}$ population was found to possess a 70 -ns decay time. ${ }^{10}$ These results are in contrast to findings in crystalline materials, where one finds that the elementary anharmonic decay processes speed up instead of slow down toward higher frequencies. To explain these remarkable experimental results, we proposed the localization of high-frequency phonons in amorphous silicon. In the present paper, we will bear out this explanation by performing a quantitative analysis using the fracton model. We will show that this model yields anharmonic decay times that fit our data very well using a realistic value for the third-order anharmonic coupling constant. First, however, we will present some experimental results that allow for a substantially more accurate estimate of the phonon decay time in amorphous silicon than was previously feasible. ${ }^{9}$

\section{EXPERIMENTAL DETAILS}

We perform anti-Stokes Raman-scattering experiments that probe the dynamics of high-frequency $\left(100-500 \mathrm{~cm}^{-1}\right)$ phonons in amorphous silicon at liquid-helium temperature. The phonons are created by fast relaxation of hot charge carriers, following pulsed laser excitation. ${ }^{11}$ These phonons are detected by means of anti-Stokes Raman scattering. This technique is particularly suitable for amorphous materials, since their entire phonon density of states can be studied by Raman spectroscopy, instead of zero-wave-vector phonons only, as in crystals. ${ }^{12}$

A $1-\mu \mathrm{m}$-thick pure $a$-Si layer was produced by implanting $1-\mathrm{MeV}^{28} \mathrm{Si}$ ions into crystalline silicon held at $77 \mathrm{~K}$ up to a dose of $3 \times 10^{15} / \mathrm{cm}^{2}$. For the experiments, the sample 


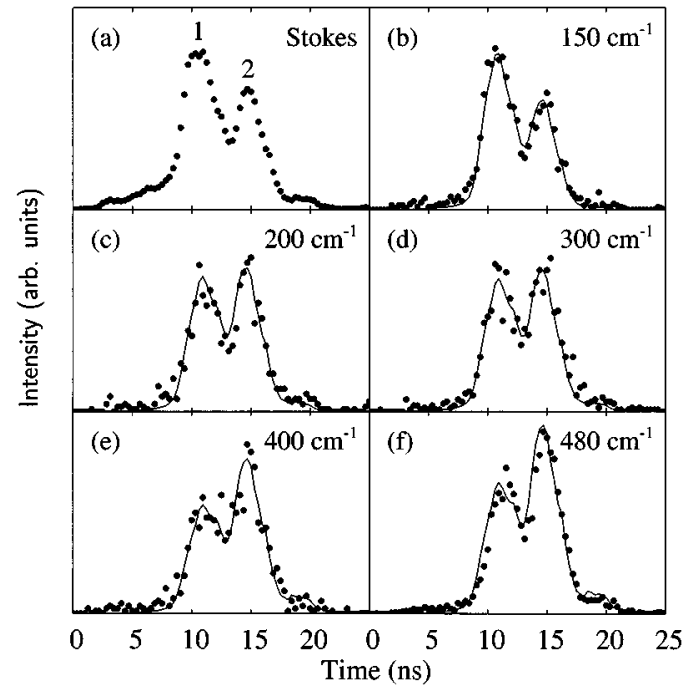

FIG. 1. Time dependence of Stokes and anti-Stokes Raman signals in $a$-Si. (a) Stokes signal. (b)-(f) Dots are the anti-Stokes time traces for phonon frequencies $\hbar \omega=150,200,300,400$, and 480 $\mathrm{cm}^{-1}$. Solid lines are fits to the data, described in the text. For the magnitude of the Stokes and anti-Stokes signals we refer to Ref. 9.

was mounted immersed in pumped liquid helium $(2 \mathrm{~K})$ in a cryostat with optical access.

A frequency-doubled, $Q$-switched Nd:YAG (yttrium aluminum garnet) laser is used to produce 532-nm pulses of 10 -ns duration at a repetition rate of $30 \mathrm{~Hz}$. The pulse-topulse fluctuations of the laser are minimized by carefully aligning the cavity, as checked by an oscilloscope. The laser beam is weakly focused to a spot of approximately $1 \mathrm{~mm}^{2}$ on the sample. The penetration depth of the green light is $\sim 100 \mathrm{~nm}$ in these samples.

In a backscattering geometry, light scattered from the sample is collected and analyzed by a double monochromator (spectral slit width $14 \mathrm{~cm}^{-1}$ ). Photons are counted by means of a fast, Peltier-cooled photomultiplier tube. A timeto-amplitude converter (TAC) is used to track the signals in time with a resolution of $1 \mathrm{~ns}$. If needed, the detected light is attenuated to less than 3 counts/s to ensure proper timeresolved single-photon counting.

\section{EXPERIMENTAL RESULTS AND ANALYSIS}

To study the temporal evolution of the optically generated phonon distribution, the Stokes and anti-Stokes signals are measured vs time. Figure 1(a) shows the Stokes Raman signal in $a$-Si vs time. We find the same shape for all phonon frequencies, and observe that it simply tracks the laser pulse. It consists of two well-separated pulse components and corresponds to mode beating of the laser. We label them 1 and 2 . In Figs. 1(b)-1(f) the anti-Stokes Raman signals are presented vs time for phonon frequencies $\hbar \omega=150,200,300$, 400 , and $480 \mathrm{~cm}^{-1}$, respectively. Here it is observed that the time dependence of the anti-Stokes Raman signals depends markedly on the phonon frequency. For the $150-\mathrm{cm}^{-1}$ vibrations, for example, the first pulse component is stronger than the second, as in the Stokes trace of Fig. 1(a). However, for the 200- and $300-\mathrm{cm}^{-1}$ vibrations, the two pulse components have comparable strengths. Even more strikingly, for

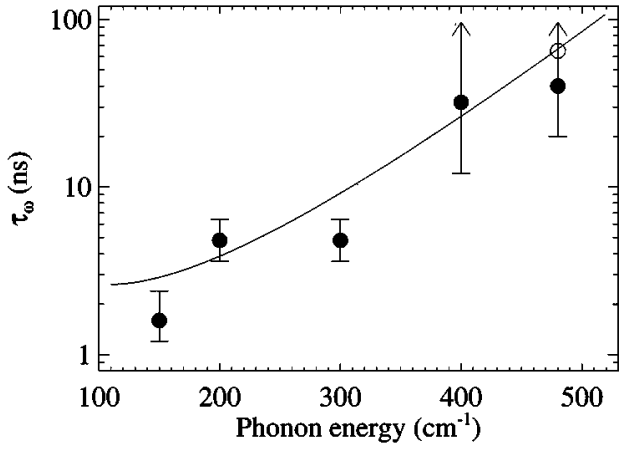

FIG. 2. Fitted values of $\tau_{\omega}$ vs phonon energy, obtained from the fits of Fig. 1 (filled circles). The open circle is the result of a separate measurement in $a$-Si:H (Ref. 10). The arrows at the error bars indicate infinity. The solid line is the result of a calculation based on the fracton model.

$\hbar \omega=400$ and $480 \mathrm{~cm}^{-1}$ the second pulse component is the largest.

At this point we wish to extract phonon decay times from the data of Fig. 1. We assume an exponential phonon population decay for phonons of energy $\hbar \omega$, characterized by decay time $\tau_{\omega}$. The anti-Stokes signal $I_{\mathrm{AS}}(t)$ is now given by $^{9}$

$$
I_{\mathrm{AS}}(t) \propto h(t) \int_{0}^{t} h\left(t^{\prime}\right) \exp \left(-\frac{t-t^{\prime}}{\tau_{\omega}}\right) d t^{\prime} .
$$

For $h(t)$, we simply take the measured Stokes signal. The solid lines in Fig. 1 are then found by fitting Eq. (1) to the data.

The resulting fitting parameter $\tau_{\omega}$, including its uncertainty, is shown vs frequency in Fig. 2 as filled circles dressed with error bars. The open circle is the result obtained for a separate measurement in $a-\mathrm{Si}: \mathrm{H}$, described in Ref. 10, and has an inherently higher precision. Of course, the value of the decay time is most reliable in the regime where $1 \mathrm{~ns}$ $\lesssim \tau_{\omega} \lesssim 10$ ns, i.e., where the lower and upper limits are determined by the time resolution of the photon counting and the laser-pulse width, respectively.

We note that our earlier measurements ${ }^{9}$ suffered from disturbing pulse-to-pulse fluctuations to a degree that an analysis such as the present one was impossible. They did, however, show the enhancement of $\tau_{\omega}$ toward higher frequencies.

\section{FRACTON MODEL}

In this section, we will demonstrate how calculations, based on the fracton model, yield elementary anharmonic lifetimes of high-energy phonons that compare favorably to the present experimental results. It will be shown that the enhancement of phonon lifetimes toward higher energies follows naturally as a consequence of the increasing localization of phonons.

Although some authors have claimed that amorphous silicon has fractal properties, ${ }^{13}$ it is generally assumed that amorphous silicon is not a fractal material. Indeed, there is no consensus in the literature about the applicability of fracton-model results to amorphous materials in general. 
Still, we believe that a comparison with the fracton model is justified, because the most pronounced common property of fractals and amorphous silicon is the absence of translational symmetry. In any case, the model is, as far as we know, the only theory that treats anharmonic interaction of vibrations above the Ioffe-Regel limit rigorously.

In the fracton model, a crossover frequency or mobility edge $\omega_{c}$ figures, below which phonons are extended. The mean free path of these extended phonons decreases rapidly for increasing frequency, until the Ioffe-Regel limit $\omega \tau(\omega) \sim 1$ is reached, with $\tau(\omega)$ the phonon lifetime. Above this limit, in this model coinciding with phonon mobility edge $\omega_{c}$, the vibrations are called fractons. The fracton wave functions are localized with a localization length $l_{\omega}$ given by

$$
l_{\omega}=\frac{\xi}{2}\left(\frac{\omega_{c}}{\omega}\right)^{\bar{d} / D}
$$

Here $\xi$ is the characteristic crossover length, a material parameter that may be identified with the length scale of medium range order in amorphous materials, ${ }^{14}$ and approximated by $2 \pi v_{s} / \omega_{c}$, the wavelength of a phonon residing at the Ioffe-Regel limit. Here $v_{s}$ denotes the sound velocity. $D$ is the so-called fractal or Hausdorff dimension, which we will take for amorphous silicon equal to the Euclidean dimension $(d=3)$, and $\overline{\bar{d}}$ is the fracton or spectral dimension of the network that must be $\leqslant 2$ for the wave functions to localize.

According to the fracton model, the prominent anharmonic decay process for fractons above the mobility edge is their tunneling to a nearby fracton state with an energy difference sufficiently small that it can be taken up by a lowfrequency extended phonon. For low temperatures, $k_{B} T \ll \hbar \omega_{c}$, the rate of this process is ${ }^{15}$

$$
\frac{1}{\tau(\omega)}=\frac{16 \pi^{3} C_{\mathrm{eff}}^{2} \Omega}{\rho^{3} v_{s}^{2} \xi^{7} \omega_{c}^{3}}\left(\frac{\omega}{\omega_{c}}\right)^{4 \overline{\bar{d}} / D+\overline{\bar{d}}-3} \hbar \omega_{c} \exp \left[-\left(\frac{\omega}{\omega_{c}}\right)^{d_{\phi} / D}\right] .
$$

Here $C_{\text {eff }}$ is the effective anharmonic coupling constant, $\rho$ is the mass density, and $\Omega=2 \pi^{3 / 2} / \Gamma(3 / 2)=12.5$. Note that the $\omega$ dependence of the phonon lifetime is $\omega^{-4 \overline{\bar{d}} / D-\overline{\bar{d}}+3} \exp \left[\left(\omega / \omega_{c}\right)^{d_{\phi} / D}\right]$, instead of the familiar $\omega^{-5}$ dependence in crystals. The exponential factor reflects the increasing localization of the fracton wave functions toward higher frequencies [see Eq. (1)], and the reduction of the fracton hopping rate by the limited wave-function overlap of the two fractons involved. For the case in point, it even diminishes towards higher $\omega$, for $\omega \gtrsim 1.7 \omega_{c}$.

The evaluation of Eq. (3) is subject to a great deal of uncertainty in the parameters involved. There are, in fact, many ways to fit this equation to the data of Fig. 2. We will discuss the worst-case scenario $\overline{\bar{d}}=2$, corresponding to the situation that the wave functions are at the edge of localization. Then Eq. (3) reduces to

$$
\frac{1}{\tau(\omega)}=P\left(\frac{\omega}{\omega_{c}}\right)^{5 / 3} \exp \left[-\left(\frac{\omega}{\omega_{c}}\right)^{d_{\phi} / D}\right]
$$

The prefactor $P$ is given by

$$
P=\frac{16 \pi^{3} C_{\mathrm{eff}}^{2} \Omega \hbar}{\rho^{3} v_{s}^{2} \xi^{7} \omega_{c}^{2}}
$$

and equals $1 / e$ times the value of the fracton hopping rate at $\omega=\omega_{c}$ [see Eq. (4)]. Note, however, that for $\omega<2 \omega_{c}$ the fracton-fracton decay process will be dominated by the much stronger process of fracton breakup into two extended phonons.

We will now estimate the value of $\omega_{c}$. We extrapolate the experimental result that the mean free path against elastic scattering of $29-\mathrm{cm}^{-1}$ phonons in $a-\mathrm{Si}: \mathrm{H}$ is $\sim 70 \mathrm{~nm}$, approximately 12 times their wavelength. ${ }^{16}$ Assuming an $\omega^{4}$ scattering law and a linear dispersion relation, we find that the Ioffe-Regel limit is reached at an energy $\hbar \omega_{c}$ $\approx \sqrt[3]{12} \times 29 \mathrm{~cm}^{-1}=65 \mathrm{~cm}^{-1}$, corresponding to a frequency of about $2 \mathrm{THz}$.

Equation (4) is now fitted to the data. The result is displayed in Fig. 2 as the solid line. The fitting procedure yields $d_{\phi}=3$, and $P=0.9 \times 10^{9} \mathrm{~s}^{-1}$. Many other choices of $\omega_{c}, \overline{\bar{d}}$, and $d_{\phi} / D$ give fits of comparable quality. The value of $P$, however, remains of the same order of magnitude. We demonstrate now that the fitted value for $P$ is indeed a physically reasonable one. Using Eq. (5), combined with the approximation $\xi \approx 2 \pi v_{s} / \omega_{c}$, for the effective third-order anharmonic coupling constant we find

$$
C_{\mathrm{eff}} \approx\left(\frac{P \rho^{3} v_{s}^{9}}{2 \Omega h \nu_{c}^{5}}\right)^{1 / 2}
$$

Substituting $\rho=2300 \mathrm{~kg} / \mathrm{m}^{3}, \quad v_{s}=5000 \mathrm{~m} / \mathrm{s}$, and $\nu_{c}$ $=\omega_{c} /(2 \pi)=1.95 \mathrm{THz}$, we arrive at $C_{\text {eff }} \approx 2 \times 10^{11} \mathrm{~J} / \mathrm{m}^{3}$. This value of $C_{\text {eff }}$ is indeed of the right order of magnitude for an anharmonic coupling constant, and is surprisingly close to the $c$-Si third-order elastic constants. ${ }^{17}$

The calculation shows that high-frequency phonon lifetimes can be severely elongated in the case of localization. More importantly, the enhancement of the phonon lifetime toward higher frequencies follows naturally in this model as a consequence of the decreasing spatial extent of the phonons toward higher energies. Finally, we note that the distribution of decay times of $480-\mathrm{cm}^{-1}$ phonons ${ }^{10}$ is quite understandable in this context: the decay mechanism described above involves a distribution in hopping distances, and thus a distribution of hopping probabilities.

\section{DISCUSSION}

As we have seen in Sec. IV, our experimental data on the slowing down of the phonon decay towards higher frequencies agree very well with the predictions of the fracton model, suggesting that anharmonic decay is the limiting process in our experiments and slows down considerably toward higher frequencies. However, as mentioned in Sec. I the assumption of phonon localization in amorphous silicon is at variance with computer models ${ }^{6,7}$ that predict Anderson localization for only a few percent of the states at the upper edge of the phonon spectrum. A limitation of these models is, however, that two-level systems, soft modes, voids, and other defects that give rise to severe phonon scattering are not included. Moreover, computer simulations may, in principle, suffer from finite-size effects. At present, the model of 
Ref. 7 is restricted to harmonic spatial diffusion of highfrequency phonons in amorphous silicon, the effect of which can be shown to be sufficiently slow to be negligible in the geometry of our experiment. ${ }^{16}$ It is very important to learn what these models predict for the anharmonic decay of highfrequency phonons. We look forward to the results of simulations presently in preparation. ${ }^{18}$

\section{ACKNOWLEDGMENTS}

We thank F. J. M. Wollenberg and C. R. de Kok for valuable technical assistance, and P. A. Stolk (AMOLF Institute) for providing the $a-\mathrm{Si}$ samples. It is a pleasure to acknowledge the fruitful collaboration with Dr. A. V. Akimov. We thank Professor R. Orbach for many helpful discussions.
${ }^{1}$ R.C. Zeller and R.O. Pohl, Phys. Rev. B 4, 2029 (1971).

${ }^{2}$ For a review, see W. A. Phillips, Rep. Prog. Phys. 50, 1657 (1987).

${ }^{3}$ For a review, see Y. M. Galperin, V. G. Karpov, and V. I. Kozub, Adv. Phys. 38, 669 (1989).

${ }^{4}$ S. Alexander, C. Laermans, R. Orbach, and H. M. Rosenberg, Phys. Rev. B 28, 4615 (1983); J. E. Graebner, B. Golding, and L. C. Allen, ibid. 34, 5696 (1986).

${ }^{5}$ A. Jagannathan, R. Orbach, and O. Entin-Wohlman, Phys. Rev. B 39, 13465 (1989).

${ }^{6}$ R. Biswas, A. M. Bouchard, W. A. Kamitakahara, G. S. Grest, and C. M. Soukoulis, Phys. Rev. Lett. 60, 2280 (1988).

${ }^{7}$ J. L. Feldman, M. D. Kluge, P. B. Allen, and F. Wooten, Phys. Rev. B 48, 12589 (1993); P. B. Allen and J. P. Feldman, ibid. 48, 12581 (1993).
${ }^{8}$ P. Sheng, M. Zhou, and Z. Q. Zhang, Phys. Rev. Lett. 72, 234 (1994); P. Sheng and M. Zhou, Science 253, 539 (1991).

${ }^{9}$ A. J. Scholten, A. V. Akimov, and J. I. Dijkhuis, Phys. Rev. B 47, 13910 (1993).

${ }^{10}$ A. J. Scholten, A. V. Akimov, P. A. W. E. Verleg, J. I. Dijkhuis, and R. S. Meltzer, J. Non-Cryst. Solids 164-166, 923 (1993).

${ }^{11}$ Z. Vardeny and J. Tauc, Phys. Rev. Lett. 46, 1223 (1981).

${ }^{12}$ M. H. Brodsky, in Light Scattering in Solids, edited by M. Cardona (Springer-Verlag, Berlin, 1975), p. 205.

${ }^{13}$ M. Ivanda, Phys. Rev. B 46, 14893 (1992).

${ }^{14}$ S. R. Elliott, Europhys. Lett. 19, 201 (1992).

${ }^{15}$ R. Orbach and A. Jagannathan, J. Phys. Chem. 98, 7411 (1994).

${ }^{16}$ A. J. Scholten, Ph.D. thesis, University of Utrecht, 1995.

${ }^{17}$ S. Tamura, Phys. Rev. B 31, 2574 (1985).

${ }^{18}$ P. B. Allen and J. L. Feldman (private communication). 